participate. 'Parents were also informed that children who underwent venepuncture would be given a T-shirt bearing the study's initials and an illustration of healthy lifestyle as a token of our appreciation of the child's participation and cooperation in the study'. This implies that a bribe was offered in advance. For research to be 'ethical', a 'token' reward should not be part of the negotiations towards recruitment for the study and should not be contingent on the child completing participation.

The authors conclude that 'our recommendations have to be scrutinised in the context of recent guidelines on the conduct of research in children by the Medical Research Council and British Paediatric Association'. ${ }^{3}$ Although the strict legality of what is usually called 'nontherapeutic research' remains uncertain, it is generally accepted that such research is ethical, provided that certain conditions are met. Assuming the consent of parents and the approval of an ethics committee, all such projects also involve a value judgment in deciding what is an appropriate balancing of the potential harms to the individual child participants with the potential gains for other children. In the example under discussion, the value of the information obtained must be set against the pain and distress caused by the venepuncture. In these circumstances a venepuncture seemed to be acceptable to the BPA Ethics Advisory Committee in 1980.4 In 1985 , a study of 92 healthy children aged between 6 and 8 years who had a venepuncture, and were followed up by questionnaire 18 months later, revealed few negative effects, and in some cases positive effects. ${ }^{5}$ Surprisingly, in the current BPA guidelines, venepuncture is classified as 'low' rather than 'minimal' risk, and it is stated that it would be unethical to submit child subjects to more than minimal risk when the procedure offers no benefit to them'. However, as the BPA President points out in an accompanying commentary, classifying venepuncture as 'low risk' might discourage important research that could benefit children generally. He emphasises that every effort should be made 'to reduce the amount of pain suffered by children by performing procedures skilfully and by considering the use of anaesthetic agents'. This recommendation was followed by Hammond et al. Perhaps the Ethics Advisory Committee might consider reclassifying venepuncture as 'minimal risk' provided that an anaesthetic agent is used to minimise pain.

The authors mention 'recommendations'. What recommendations? I was unable to discover any advice that might be useful to others in carrying out similar studies. For example, it would have been useful to have the benefit of their experience in suggesting ways of overcoming the poor response rate from schools with children from deprived social backgrounds and of minimising the number of children who dissent at the time of venepuncture. It would also have been interesting to know why the third phlebotomist had such a significant failure rate. For example, assuming basic technical expertise, how important was the personality of the phlebotomist and the ability to communicate effectively with apprehensive children and parents - so important in a project of this kind?

A G M CAMPBELL 34 Woodburn Crescent, Aberdeen $A B 187 X$

1 Beecher HK. Ethics and clinical research. $N$ Engl $f$ Med 1966; 274: 1354-60.

2 Medical Research Council. Working party on research in children. The ethical conduct of research on children. London: MRC, 1991. (MRC Ethics Series.)

3 British Paediatric Association. Guidelines for the ethical conduct of medical research involving children. London: BPA, 1992. 4 British Paediatric Association. Guidelines to aid ethical committees considering research involving children. Arch Dis Child 1980; 55: 75-7.

5 Smith $M$. Taking blood from children causes no more than minimal harm. F Med Ethics 1985; 11: 127-31.

\section{Commentary (3)}

Parents, children and their doctors have, rightly, high expectations for safety in the practice of childhood medicine. The risk/ benefit ratio for practical procedures and treatments should be known so that consent might be given. Seasoned paediatricians know that the intuitive acceptability tests which parents apply are probably set at a higher standard for their children than for themselves. In research and development the traditional approach has been to try out the procedure or drug on adults and extend it to children if this experience is satisfactory. Practical and financial considerations probably explain why many drugs prescribed by paediatricians are not recommended by manufacturers for use in children; studies required to satisfy licensing bodies would be too demanding.

Two emerging arguments have a bearing here: first, the recognition of childhood autonomy, especially following the Children Act of 1989. This means that the child must be consulted and his or her decision must have an important influence on medical activities. The second is that tests and drugs should be evaluated in children as rigorously as happens with adults - and the if it works on adults try it on children' approach should be abandoned. Both of these issues have important implications on research in children, both therapeutic and, particularly, non-therapeutic. The BPA has addressed these by publishing guidelines for the ethical conduct of medical research in children, the latest edition of which appeared in 1992. These caused alarm to many of us researchers because they estimated the risk to children of injections and venepuncture as being low (rather than minimal - the least possible) and then declared it would be unethical to submit children to more than minimal risk in non-therapeutic research. This was a change from the guidelines issued in 1980 where the calculus of risk estimate was slightly different but a negligible risk was thought to be one less than was run in everyday life - and this clearly applies to blood sampling. Many researchers now fear that research ethics committees would ban non-therapeutic research involving injections, fingerpricks, or venepuncture. As a consequence, the desirable aim of extending the range and safety of procedures 
in drugs for the treatment of children, as well as vital epidemiological research, might be undermined. Put simply, the BPA Ethics Advisory Committee was in favour of the absolute principle of defending the integrity of the child rather than the utilitarian argument that minor harm to the child is justified by the benefits it would give children as a whole.

The Ethics Advisory Committee seemed persuaded by the frightening effects of needles on children, a view which seemed unbalanced to many who argue that the risk is minimal provided skilful and experienced staff provide adequate explanation and reassurance to both child and parents and use local anaesthetic creams. Happily the paper from Hammond and her colleagues supports this: there were very few refusals by parents or children - and note that children themselves were required to agree to the procedure - and the study was conducted according to the highest possible standards. It would have been interesting to know the teachers' views, especially a few days after the venepuncture sessions when they may have received some feedback from the children. Some might question the propriety of giving a present to those children who underwent the procedure - adults receive expenses but no reward. It is alarming that one phlebotomist failed to obtain samples in about $20 \%$ of children. Those responsible for such studies should be monitoring performance and intervening early with retraining or redeployment in such instances. To encourage greater participation then more parental education will be needed, particularly concerning young children and those parts of society disinclined to participate. Their under-representation is regrettable as they and their peers may not benefit from the results of selective research.

The authors are to be congratulated on carrying out this study - and the local ethics committee for agreeing to it. Armed with the BPA guidelines and Sir David Hull's excellent introductory commentary, research ethics committees should take a permissive view about non-therapeutic research in children which involves blood sampling.

The community paediatric service in Canterbury deserves a special pat on the back; such studies are not easy to organise and support and their efforts will be warmly appreciated by those who recognise the huge potential for research in community paediatrics - research of the most challenging sort because it is done on free-range children (to use the felicitous phrase of Professor J D Baum) in their own environment rather than in the more contained and controlled world of the laboratory.

T L CHAMBERS 2 Clifton Park, Bristol BS8 3BS

Commentary (4)

The paper by Dr Hammond and her colleagues is to be welcomed, particularly in the light of the
BPA's Guidelines for the Ethical Conduct of Medical Research Involving Children in which it was indicated that venepuncture in children was a procedure which could involve more than minimal risk. ${ }^{1}$ Those working on paediatric wards are familiar with the intense dislike of 'needles' expressed by many children, even to the extent of their putting up with postoperative pain rather than accept analgesia administered by injection. On the other hand important national studies inquiring into the nutritional status of primary schoolchildren are in progress and these need biochemical measurements on blood samples obtained by venepuncture. Almost certainly more such studies will be proposed in the future.

It says much for the way this project was presented that in one of the participating schools consent for the study was given by over $90 \%$ of the parents. However, a figure of only half that at another school raises questions about the previous experiences of these parents and emphasises the importance of seeking to ensure that children and parents participating in studies of this sort can see them as worthwhile.

Prior explanation did not appear to influence the relatively small number of children who declined to take part. As in the case of studies involving adults, participation of children implies their 'informed' consent and hence appropriate understanding on their part of the aims and objectives as well as of the procedures involved. Despite the organisational difficulties, there could be advantage in involving the children's teachers in explaining the nature and the value of an investigation such as this, particularly in the context of healthy living, and this would be very much in keeping with the BPA guidelines and their emphasis on research with rather than on children.

On the question of a parental presence at the time of venepuncture, the authors are right to point out that this can raise the level of anxiety. Nevertheless, the degree of trust that there should be between research workers and their subjects in this type of study surely requires that parents receive an invitation to attend while recognising that this may make for difficulties in a few instances.

This paper has demonstrated that screening in childhood involving venepuncture is possible but far from straightforward. It has also shown that to be ethical there is a requirement for high levels of technical skill on the part of phlebotomists, communication skills to involve and enthuse the schools, their pupils and the parents, and design and logistic expertise to ensure that each child's contribution does actually reach analysis. Above all there must be a genuine sense of partnership in an activity that may be challenging but is clearly for the common good.

I COIIN NORMAND 23 St Thomas' Street, Winchester,

Hampshire SO23 9HF

1 British Paediatric Association. Guidelines for the ethical conduct of medical research involving children. London: BPA, 1992. 